\title{
Construção de instrumento para anamnese e exame físico de crianças hospitalizadas: um relato de experiência
}

\author{
Construction of instrument for anamnesis and physical examination of hospitalized \\ children: an experience report
}
Construcción de instrumentos para análisis y examen físico de niños hospitalizados: un informe de experiencia

Francini de Oliveira Rodrigues ${ }^{1}$, Letícia Martins Machado ${ }^{1}$, Claudete Moreschi ${ }^{1}$, Mylena Flores Chaves $^{1}$, Bárbara Belmonte Bedin ${ }^{1 *}$.

\section{RESUMO}

Objetivo: Descrever experiência acadêmica na construção de instrumento para realizar anamnese e exame físico de crianças hospitalizadas. Relato de experiência: Trata-se de um relato de experiência acadêmica na construção de instrumentos para anamnese e exame físico voltado a elaboração do histórico de enfermagem de crianças hospitalizadas durante as atividades da disciplina de Estágio Supervisionado II, entre os meses de agosto a novembro de 2017. Foram construídos três instrumentos diferentes para avaliação da criança hospitalizada. Os instrumentos construídos foram elaborados por faixa etária devido a criança apresentar particularidades em cada uma delas. Considerações finais: A construção dos instrumentos fortaleceu a importância do processo de enfermagem para a autonomia do enfermeiro. A aplicabilidade dos instrumentos contribuirá para a qualidade do cuidado prestado à criança, bem como para a reflexão do enfermeiro acerca da busca pelo conhecimento das especificidades de cada criança durante a aplicação da Sistematização da Assistência de Enfermagem na pediatria.

Palavras-chave: Enfermagem, Criança hospitalizada, Processo de enfermagem, Enfermagem no consultório.

\begin{abstract}
Objective: To describe academic experience in the construction of an instrument to perform anamnesis and physical examination of hospitalized children. Experience report: It is an account of academic experience in the construction of instruments for anamnesis and physical examination aimed at the elaboration of the nursing history of hospitalized children during the activities of the Supervised Internship II discipline, from August to November 2017. Were built three instruments for assessing hospitalized children. The instruments were elaborated by age group due to the child presenting particularities in each one. Final considerations: The construction of instruments strengthened the importance of the nursing process for nurses' autonomy. The applicability of the instruments will contribute to the quality of care provided to the child, as well as to the nurse's reflection about the search for knowledge of the specificities of each child during the application of the Nursing Care Systematization in pediatrics.
\end{abstract}

Keywords: Nursing, Hospitalized child, Nursing process, Office nursing.

${ }^{1}$ Universidade Regional Integrada do Alto Uruguai e das Missões (URI), Santiago - RS.

*E-mail: barbarabbedin@hotmail.com

SUBMETIDO EM: $3 / 2020$

ACEITO EM: 4/2020

PUBLICADO EM: 5/2020

REAS/EJCH | Vol.Sup.n.49 | e3348 | DOI: https://doi.org/10.25248/reas.e3348.2020 Página 1 de 7 


\section{RESUMEN}

Objetivo: Describir la experiencia académica en la construcción de un instrumento para realizar anamnesis y examen físico de niños hospitalizados. Informe de experiencia: Es un relato de la experiencia académica en la construcción de instrumentos para la anamnesis y el examen físico dirigido a la elaboración de la historia de enfermería de los niños hospitalizados durante las actividades de pasantía supervisada, de agosto a noviembre de 2017. Se construyeron tres diferentes instrumentos para evaluar niños hospitalizados. Los instrumentos construidos fueron elaborados por grupo de edad debido a que el niño presenta particularidades en cada uno. Consideraciones finales: La construcción de los instrumentos fortaleció la importancia del proceso de enfermería para la autonomía de la enfermera. La aplicabilidad de los instrumentos contribuirá a la calidad de la atención brindada al niño, así como a la reflexión de la enfermera sobre la búsqueda del conocimiento de las especificidades de cada niño durante la aplicación de la Sistematización de la Atención de Enfermería en pediatría.

Palabras clave: Enfermería, Niño hospitalizado, Proceso de enfermería, Enfermería en la oficina.

\section{INTRODUÇÃO}

A enfermagem surgiu no Brasil no século XIX com a primeira escola Profissional para Enfermeiros e Enfermeiras, com intuito de formar profissionais de saúde a fim de cuidar dos enfermos (SIOBAN N, 2011). A enfermagem tem suas singularidades em cada contexto que se desenvolve e adquire características próprias ao atender as necessidades de saúde, que são reconhecidas pelo propósito que se busca conformar ou está conformado (PEREIRA MJB, et al., 2009).

O Processo de Enfermagem (PE) é um instrumento que facilita o atendimento das necessidades individuais do paciente. Também contribui para o planejamento de ações que visam o bem-estar, e a qualidade de vida do ser humano, bem como tornar o saber e o fazer da enfermagem como profissão por meio de uma linguagem padronizada e universal (MARINELLI NP, et al., 2015).

A Sistematização da Assistência de Enfermagem (AS) é uma atividade privativa do enfermeiro, que organiza o trabalho em enfermagem quanto ao método, pessoal e instrumentos, possibilitando a operacionalização do PE. O PE deve ser desenvolvido sistematicamente em todos os locais, públicos ou privados, onde ocorra o cuidado profissional de enfermagem. Além disso, organiza-se em cinco etapas: Histórico de Enfermagem, Diagnóstico de Enfermagem, Planejamento de Enfermagem, Implementação de Enfermagem e a Avaliação de Enfermagem, as quais são inter-relacionadas, interdependentes e recorrentes (COFEN, 2009; GUTIÉRREZ MGR e MORAIS SCRV, 2017).

O histórico de enfermagem é um processo deliberado, sistemático e contínuo realizado com ajuda de métodos e de técnicas diferentes (COFEN, 2009) Constitui-se pela entrevista que investiga a situação de saúde do paciente/cliente identificando os problemas e necessidades de intervenções. Para a sua elaboração, segue-se os seguintes passos: coleta de dados, identificação de padrões e comunicação e registro dos dados obtidos (SANTOS MAP, et al., 2017)

Os estudos trazem novos desafios para o enfermeiro, em especial quanto a enfermagem voltada para a saúde da criança que deve estar pautada na ética e bioética. Assim, destaca-se a importância do conhecimento científico sobre o cuidado a criança hospitalizada, bem como também para a família, pois orienta o serviço de enfermagem e promove a autonomia profissional (COSTA FM, et al., 2014; ANDRADE RC, et al., 2015).

Dentre os contextos em que há o desenvolvimento do PE, cita-se a unidade pediátrica, que se caracteriza como um espaço que a criança se encontra hospitalizada, devido algum acometimento patológico. Este ambiente deve ser planejado, organizado quanto a sua estrutura física e acolhedor, resolutivo e apoiado na ética e bioética, bem como o perfil dos profissionais voltado para o cuidado específico da criança (RIBEIRO JP, et al., 2017). 
Destaca-se que a assistência da criança hospitalizada deve estar voltada para adoção de uma abordagem profissional apoiada na ética, na qual devem ser consideradas suas individualidades e especificidades nesta etapa da vida. A Constituição da República do Brasil de 1988, "garante às crianças e adolescentes, com absoluta prioridade, o direito à vida, saúde, dignidade, respeito, liberdade [...]". Já em 1990, o Estatuto da Criança e do Adolescente (ECA) instituiu a Lei 8069, a qual proporciona que a criança possa ser vista como um ser humano completo (RODRIGUES BMRD, et al., 2014).

Ressalta-se que apesar dos benefícios da implantação da SAE no ambiente hospitalar e ser um exercício privativo do enfermeiro, esta, ainda, se considera como um desafio para os enfermeiros. Como exemplo, estudo realizado com profissionais de enfermagem, evidenciou que o processo de implantação da SAE em unidade pediátrica pode enfrentar barreiras, como a sobrecarga de trabalho dos enfermeiros, a falta de conhecimento dos técnicos de enfermagem sobre a SAE e o pouco envolvimento dos profissionais da equipe nesse processo (TAVARES TS, et al., 2013).

Nessa perspectiva, a implantação da SAE na pediatria contribui para a avaliação adequada da criança, na identificação precoce de possíveis alterações, na sua recuperação, qualidade de vida, o vínculo entre a equipe de enfermagem e a criança-família. Soma-se a isso, a importância da SAE no ambiente hospitalar, para a autonomia e reconhecimento profissional do enfermeiro à medida que desenvolverá uma competência privativa do mesmo. Desta forma, descrever experiência acadêmica na construção de um instrumento para realizar anamnese e exame físico de crianças hospitalizadas.

\section{RELATO DE EXPERIÊNCIA}

Consiste em um relato de experiência acadêmica na construção de material para a elaboração de anamnese e exame físico de crianças hospitalizadas. Acadêmicas do X semestre do curso de Graduação em Enfermagem da Universidade Regional Integrada do Alto Uruguai e das Missões localizada na cidade de Santiago, Rio Grande do Sul receberam a proposta de construir um instrumento para levantamento de dados para realizar anamnese e exame físico em uma unidade pediátrica.

A proposta emergiu da necessidade do hospital, localizado na mesma cidade, onde um grupo de cinco acadêmicas desenvolviam as atividades da disciplina de Estágio Supervisionado II, entre os meses de agosto a novembro de 2017. Para a construção do instrumento, as acadêmicas reuniram-se, semanalmente, nas dependências da universidade entre os meses de agosto e setembro de 2017.

Inicialmente, realizaram-se estudos de referências que tratam do cuidado pediátrico, como: exame físico, abordagem a criança e seus familiares a nível hospitalar, bem como discussão acerca das especificidades de cada faixa etária (BRASIL, 2013; MOREIRA MDS e GAÍVA MAM 2017). Após estudos, foram construídos instrumentos para cada faixa etária, como: 0 a 2 anos incompletos, 2 a 5 anos incompletos, e de 5 a 10 anos. Foi realizado um teste piloto do instrumento na pediatria, com o objetivo de verificar as inconsistências.

Após o processo de construção e aplicação do teste piloto, foi agendado uma reunião com as enfermeiras do hospital através da coordenação de enfermagem do hospital, com o propósito de apresentar os resultados e inconsistências do instrumento, bem como responder aos questionamentos relacionados ao mesmo.

Foram apresentados os resultados do instrumento através de slides no Microsoft PowerPoint, e orientadas quanto à importância do mesmo para avaliação específica da criança. Os instrumentos foram disponibilizados para o serviço de forma digital e estão sendo utilizados pelos enfermeiros do serviço de forma impressa.

Foram construídos três instrumentos diferentes para avaliação da criança, pois a partir da busca na literatura específica acerca da saúde da criança, identificou-se que para cada faixa etária existem avaliações diferentes e específicas para cada período de desenvolvimento da criança. Os instrumentos construídos foram para crianças de 0-2 anos de idade (incompletos); 2-5 anos de idade (incompletos); e de 5-10 anos de idade, conforme Quadro 1. 
Quadro 1 - Itens avaliados nos instrumentos, por dimensão, em cada faixa etária.

\begin{tabular}{|c|c|c|c|}
\hline \multirow{3}{*}{ Dimensões } & \multicolumn{3}{|c|}{ Faixas Etárias } \\
\hline & $0-2$ anos & 2-5 anos & $5-10$ anos \\
\hline & \multicolumn{3}{|c|}{ Itens avaliados em cada dimensão } \\
\hline Dados de identificação & \multicolumn{3}{|c|}{$\begin{array}{l}\text { Nome da criança; Idade; Data de nascimento; Naturalidade; Sexo; Com quem reside; Crença/espiritualidade da família; } \\
\text { Acompanhante; Grau de parentesco; Escolaridade; Endereço; Unidade Básica de Saúde de referência. }\end{array}$} \\
\hline Histórico clínico & $\begin{array}{l}\text { Procedência; Motivo da internação; Queixa } \\
\text { principal; Fatores de riscos; Doenças crônicas; } \\
\text { Cirurgias anteriores; Uso de medicações; Uso de } \\
\text { dispositivos invasivos; Alergias, Dieta; História } \\
\text { alimentar; Ingesta hídrica; Movimento no leito; } \\
\text { Deambulação; Imunizações. }\end{array}$ & $\begin{array}{l}\text { Além dos itens avaliados no histórico clínico da } \\
\text { faixa etária de 0-2 anos, enfatiza-se: Número de } \\
\text { refeições diárias; Aceitação da dieta; Modo de } \\
\text { preparo e preferências alimentares. }\end{array}$ & $\begin{array}{l}\text { Além dos itens } \\
\text { avaliados no histórico } \\
\text { clínico da faixa etária de } \\
\text { 0-2 anos, enfatiza-se: } \\
\text { Sono e repouso. }\end{array}$ \\
\hline $\begin{array}{l}\text { Avaliação das } \\
\text { necessidades } \\
\text { psicossociais }\end{array}$ & $\begin{array}{l}\text { Segurança; Comunicação; Lazer; Recreação e } \\
\text { interação social. }\end{array}$ & $\begin{array}{l}\text { Além dos itens avaliados na avaliação das } \\
\text { necessidades psicossociais da faixa etária de } 0 \text { - } \\
2 \text { anos, enfatiza-se: Faz amigos com facilidade; } \\
\text { Relaciona-se bem com os amigos; Brincadeiras } \\
\text { e brinquedos preferidos; Idade das crianças que } \\
\text { brinca. }\end{array}$ & - \\
\hline $\begin{array}{l}\text { Relacionamento com os } \\
\text { pais }\end{array}$ & \multicolumn{2}{|c|}{$\begin{array}{l}\text { Pais vivem juntos; Ocupação dos pais; Realização de planejamento familiar; Local onde a criança fica } \\
\text { maior parte do tempo. }\end{array}$} & - \\
\hline Sono & - & $\begin{array}{l}\text { Dorme com os pais; Dorme com outras pessoas; } \\
\text { Quantas horas de sono diário; Qualidade do } \\
\text { sono (dorme bem, range os dentes, fala durante } \\
\text { a noite tem medo de escuro, ronca, falta de } \\
\text { ar/apnéia, grita durante a noite, sono leve, } \\
\text { acorda várias vezes). }\end{array}$ & - \\
\hline
\end{tabular}




\begin{tabular}{|c|c|c|}
\hline \multirow{3}{*}{ Dimensões } & \multicolumn{2}{|l|}{ Faixas Etárias } \\
\hline & $2-5$ anos & 5-10 anos \\
\hline & \multicolumn{2}{|l|}{ Itens avaliados em cada dimensão } \\
\hline Eliminações fisiológicas & \multicolumn{2}{|c|}{$\begin{array}{l}\text { Uso de fraldas; Eliminações urinarias; Aspectos fisiológicos e coloração da urina; Eliminações intestinais; Número e aspecto de } \\
\text { evacuações diárias. }\end{array}$} \\
\hline $\begin{array}{l}\text { Saúde da mãe durante a } \\
\text { gestação }\end{array}$ & $\begin{array}{l}\text { Planejamento de gestação; Início do pré-natal; Intercorrência durante a gestação; Tipo de parto; Idade } \\
\text { gestacional na data do parto. }\end{array}$ & - \\
\hline Condições de moradia & \multicolumn{2}{|l|}{ Tipo de casa; Saneamento básico; Pessoas que residem na casa. } \\
\hline Condições de higiene & \multicolumn{2}{|l|}{ Descrição das condições de higiene pessoal. } \\
\hline Exame físico & $\begin{array}{l}\text { Escala de Glasgow; Escala de Braden Pediátrica; Frequência Cardíaca; Frequência Respiratória; } \\
\text { Temperatura Axilar e Saturação; Avaliação da Dor; Sono e repouso; Exame físico céfalo podal. }\end{array}$ & $\begin{array}{lrr}\text { A avaliação da dor } \\
\text { ocorre por meio da } \\
\text { aplicabilidade } \\
\text { Escala } & \text { da } \\
\text { Analógica. } & \end{array}$ \\
\hline Gráficos & \multicolumn{2}{|l|}{ Gráfico para avaliação de peso e gráfico para avaliação de estatura conforme faixa etária. } \\
\hline $\begin{array}{l}\text { Diagnóstico de } \\
\text { Enfermagem }\end{array}$ & \multicolumn{2}{|l|}{ Conforme Taxonomia North American Nursing Diagnosis Association (NANDA) } \\
\hline Prescrição de Enfermagem & \multicolumn{2}{|l|}{ De acordo com os diagnósticos de enfermagem elencados. } \\
\hline Evolução de Enfermagem & \multicolumn{2}{|l|}{ Realizada diariamente. } \\
\hline
\end{tabular}

Fonte: Rodrigues FO, et al., 2017. 


\section{DISCUSSÃO}

Destaca-se que a construção dos instrumentos se deu a partir da busca de artigos relacionados ao crescimento e desenvolvimento da criança e avaliação através da consulta de enfermagem no momento que a criança encontra-se hospitalizada (COSTA FM, et al., 2014; SILVA JP, et al., 2015; LEITE TMC, et al., 2017). Para construção dos três instrumentos considerou-se a "Caderneta da Criança", a qual tem uma avaliação específica para o sexo masculino e feminino, e subdivide-se também em faixas etárias, para adequada avaliação da criança.

Nesse sentido, a criança deve passar cada estádio segundo uma sequência regular, ou seja, os estádios de desenvolvimento cognitivo são sequenciais. Assim, se a criança não for estimulada ou motivada no devido momento, ela não conseguirá superar o atraso do seu desenvolvimento, pois o desenvolvimento infantil se dá à medida que a criança vai crescendo e vai se desenvolvendo de acordo com os meios onde vive e os estímulos deles recebido (BRASIL, 2017).

Os instrumentos construídos foram elaborados por faixa etária devido a criança apresentar particularidades em cada uma delas, as diferenças acontecem conforme a estimulação e cuidado da família, escola e todas as pessoas de seu convívio. A criança de 1 a 2 meses desenvolve o predomínio do tônus flexor, assimetria postural e preensão reflexa, a percepção melhor do rosto e a distância com o seio materno; entre 2 e 3 meses o sorriso social acontece, inicia-se o processo de ampliação do campo visual, e adquire a noção de profundidade; aos 4 meses começa a preensão voluntária das mãos (BRASIL, 2012).

Além disso, em torno dos 6 meses inicia-se a "permanência do objeto", começa a apresentar reações com pessoas estranhas e engatinhar até os 9 meses; em 7 meses a criança começa a sentar sem apoio; em torno do $10^{\circ}$ mês a criança pode começar a ficar em pé; em 12 meses começa a ter acuidade visual adulta; com 2 anos de idade a criança diz seu próprio nome e dá nome aos objetos; de 4 a 6 anos de idade a memória e a habilidade com a linguagem aumentam, os ganhos cognitivos melhoram sua capacidade de tirar proveito da educação formal, sua autoimagem desenvolve-se e compreende a constância de gênero (BRASIL, 2012).

Por fim, de 7 a 9 anos a criança começa a desenvolver o julgamento global de autovalor, integrando sua autopercepção, "fechando" algumas ideias sobre quem ela é e como deve ser. A influência dos amigos e colegas da mesma idade tem importância nesta etapa da vida, enquanto a influência dos pais diminui; a partir dos 10 anos ocorrem mudanças relacionadas à puberdade e há um estirão de crescimento. Diante das mudanças que ocorrem através do estímulo da criança em cada momento de sua vida, justifica-se a construção de um instrumento que considere as especificidades de cada faixa etária (BRASIL, 2012).

Quanto a consulta de enfermagem e a sistematização, sabe-se que o enfermeiro na atenção básica faz o acompanhamento da criança por meio da consulta de puericultura desde os primeiros dias de vida, a fim de acompanhar o seu crescimento e desenvolvimento, identificar alterações e orientar a família sobre os cuidados com a mesma. No entanto, na atenção hospitalar o foco está na sintomatologia apresentada pela criança com vistas ao tratamento. Porém, mesmo, neste cenário, é importante que o enfermeiro desenvolva o $\mathrm{PE}$, pois, esta é uma ferramenta que favorece a autonomia profissional à medida que seria mais resolutivo em sua assistência (ZANARDO GM, et al., 2017).

Nessa perspectiva, compreende-se a importância da consulta de enfermagem para avaliação da criança, pois caracteriza-se como uma ação que reorganiza a assistência básica a saúde, bem como contribui em subsídios para o desenvolvimento de ações de promoção, proteção e recuperação da saúde e o tratamento de doenças (MOREIRA MDS e GAÍVA MAM, 2017).

Destaca-se que a consulta de enfermagem em puericultura objetiva o acompanhamento criterioso do crescimento e desenvolvimento da criança, engloba um conjunto de medidas de cuidados preventivos, com um olhar abrangente que cuida da criança, mãe e família, afim de compreender suas necessidades individuais (SOARES DG, et al., 2016).

A construção de um instrumento para a operacionalização do PE fortaleceu o conhecimento enquanto acadêmicas, à medida que possibilitou relembrar as etapas do processo de enfermagem e a importância 
deste para o avanço científico da autonomia profissional do enfermeiro. Construir os instrumentos e ter a oportunidade de desenvolvê-los durante a prática fez refletir sobre a importância do PE. Acredita-se que a elaboração minuciosa dos instrumentos, levando em consideração as especificidades de faixas etárias da criança, contribuirá na qualidade da assistência de enfermagem prestada a esta população. Tais instrumentos foram elaborados para serem utilizados privativamente pelos enfermeiros.

O enfermeiro responsável pela equipe de enfermagem e pela assistência do paciente deve ter conhecimento sobre o modelo de saúde que será executado, bem como habilidades e competências necessárias para o seu desenvolvimento (MARINELLI NP, et al., 2015).

Soma-se a isso, que o PE constitui uma ferramenta para gestão do cuidado, pois envolve aspectos que transcendem ao cuidado direto, possibilita a avaliação da eficiência e da eficácia de atividades realizadas, bem como contribui para tomada de decisão gerencial e política, do enfermeiro, a fim de prestar uma assistência adequada (GUTIÉRREZ MGR e MORAIS SCRV, 2017).

O estudo traz que a implantação do PE, deve ser a partir de conhecimento específico, de uma reflexão crítica, bem como de questionamentos acerca da organização do trabalho de enfermagem. A aplicabilidade da SAE é essencial para que o enfermeiro possa gerenciar e aprimorar a assistência de enfermagem de maneira organizada, segura, dinâmica e competente (MARINELLI NP, et al., 2015).

Quanto à implantação do PE na atenção hospitalar, acredita-se que poderia ser mais eficaz, quando o enfermeiro tem o suporte de toda a equipe e da instituição, que busca novos avanços científicos, valoriza o profissional, a sua opinião e aceita novas mudanças.

Estudo considera que a implantação poderia ser mais efetiva através de uma gestão participativa, na qual os profissionais de enfermagem tenham a oportunidade de compreender, construir ou reconstruir seu processo de trabalho, em conjunto com os gestores. Considera-se que um processo participativo de toda a equipe, pode romper com os movimentos centralizados, verticalizados ou fragmentados de implantação do PE (TRINDADE LR, et al., 2015).

As discussões em grupo foram consideradas significativas e importantes para construção dos instrumentos, bem como relevante na hora de aplicar o teste piloto, à medida que se realizou leituras, estudos e discussões de diferentes autores relacionados ao PE e a criança hospitalizada. Estes contribuíram para o fortalecimento enquanto acadêmicas no decorrer das práticas de estágios no âmbito hospitalar. O que confirma a importância da busca pelo conhecimento científico no desenvolvimento da prática profissional do enfermeiro.

A construção da autonomia deve se sustentar em uma prática cientificamente comprovada. $O$ desenvolvimento do PE é uma atribuição do enfermeiro, e se constitui em um instrumento importante de sustentação para sua autonomia na prática. Considera-se que enfermeiros com altos níveis de autonomia têm a responsabilidade e a oportunidade de contribuir e tomar decisões na sua assistência, o que inclui as questões políticas e pessoais que afetam o contexto do cuidado (SANTOS FOF, et al., 2012).

A autonomia é composta por duas dimensões: a dimensão pessoal ou de atitude e a dimensão estrutural. A autonomia concedida as diferentes áreas profissionais se diferem entre as instituições de saúde. Entretanto, o enfermeiro deve reconhecer as oportunidades de tomar decisões, aproveitar os espaços ofertados pelas instituições e construir caminhos em busca de autonomia (SANTOS FOF, et al., 2012).

Deste modo, destaca-se que é importante que durante a formação do profissional, sejam ofertados espaços para discussões que contribuam para a prática do enfermeiro, pois, a busca pelo conhecimento começa na formação, a qual orienta e ensina o futuro profissional, a fim de inserir o acadêmico na realidade desde a sua formação.

A elaboração dos instrumentos fortaleceu a importância do Processo de Enfermagem para a autonomia do enfermeiro. A aplicabilidade dos instrumentos contribuirá para a qualidade do cuidado prestado à criança, bem como para a reflexão do enfermeiro acerca da busca pelo conhecimento das especificidades de cada criança durante a aplicação da Sistematização da Assistência de Enfermagem na pediatria. 


\section{REFERÊNCIAS}

1. SIOBAN N. A imagem da enfermeira - As origens históricas da invisibilidade na enfermagem. Texto e Contexto, $2011 ; 20(2): 223-4$.

2. PEREIRA MJB, et al. A enfermagem no Brasil no contexto da força de trabalho em saúde: perfil e legislação. Revista Brasileira de Enfermagem, 2009; 62(5):771-7.

3. MARINELLI NP, et al. Sistematização da Assistência de Enfermagem: desafios para implantação. Revista Enfermagem Contemporânea, 2015; 4(2):254-263.

4. COFEN. Conselho Federal de Enfermagem. Resolução n. 358, de 15 de outubro de 2009.

5. GUTIÉRREZ MGR e MORAIS SCRV. Sistematização da Assistência de Enfermagem e a formação da identidade profissional. Revista Brasileira de Enfermagem. 2017; 70(2):455-60.

6. SANTOS MAP, et al. "Processo de Enfermagem" Sistematização da Assistência de Enfermagem - SAE. Saúde em Foco, 2017; 679-83.

7. COSTA FM, et al. Sistematização da Assistência de Enfermagem em uma unidade de internação pediátrica: percepção da equipe de enfermagem. Revista Norte Mineira de Enfermagem, 2014; 3(2):104-19

8. ANDRADE RC, et al. Necessidades dos pais de crianças hospitalizadas: evidências para o cuidado. Revista Eletrônica de Enfermagem, 2015; 17(2):379-94.

9. RIBEIRO JP, et al. Ambiente de pediatria: aspectos que auxiliam no processo de trabalho e na produção de saúde. Revista de Enfermagem UFPE OnLine, 2017; 11(Supl. 12):5275-81.

10. RODRIGUES BMRD, et al. A ética no cuidado à criança hospitalizada: uma perspectiva para a enfermagem. Revista de Pesquisa Cuidado é Fundamental Online, 2014; 6(4):1475-84.

11. TAVARES TS. Avaliação da implantação da Sistematização da Assistência de Enfermagem em uma unidade pediátrica. Revista Mineira de Enfermagem. 2013; 17(2): 278-86.

12. BRASIL. Ministério da Saúde. Caderneta de saúde da criança. 11a ed. Brasília: Ministério da Saúde, 2017.

13. MOREIRA MDS e GAÍVA MAM. Abordagem do contexto de vida da criança na consulta de enfermagem. Revista de Pesquisa Cuidado é Fundamental Online, 2017; 9(2): 432-40.

14. SILVA JP. Sistematização da Assistência de Enfermagem na graduação: um olhar sob o Pensamento Complexo. Revista Latino-americana de Enfermagem, 2015; 23(1):59-66.

15. LEITE TMC. Processo de trabalho do enfermeiro pediatra: uma realidade a ser transformada. Revista Rene, 2017; 18(1): 26-34.

16. BRASIL. Ministério da Saúde. Secretaria de Atenção à Saúde. Departamento de Atenção Básica. Saúde da criança: crescimento e desenvolvimento. Brasília: Ministério da Saúde, 2012.

17. ZANARDO GM, et al. Atuação do enfermeiro na consulta de puericultura: uma revisão narrativa da literatura. Revista de Enfermagem (Frederico Westphalen, Online). 2017; 13(13):55-69.

18. SOARES DG, et al. Implantação da puericultura e desafios do cuidado na Estratégia Saúde da Família em um município do estado do Ceará. Revista Brasileira em Promoção da Saúde, 2016; 29(1): 132-8.

19. TRINDADE LR, et al. Compreensão do Processo de Enfermagem por enfermeiros de um hospital geral do sul do Brasil. Revista de Enfermagem da UFSM. 2015; 5(2): 267-77.

20. SANTOS FOF, et al. Autonomia profissional e Sistematização da Assistência de Enfermagem: Percepção de Enfermeiros. Revista Mineira de Enfermagem, 2012; 16(2): 251-7. 